\title{
Effects of Orientational Relaxation of Polymer Chains Induced by Isotropic Particles on the Enhanced Thermal Conductivity of AlN-filled Polyimide Films
}

\author{
Mizuka Tanimoto and Shinji Ando \\ Department of Chemistry and Materials Science, Tokyo Institute of Technology, \\ Ookayama 2-12-1-E4-5, Meguro-ku, Tokyo 152-8552, Japan
}

\begin{abstract}
A series of composite films composed of $\mu \mathrm{m}$-sized aluminum nitride (AIN) particles and polyimides (PIs) were fabricated using (a) rigid-chain $s$ BPDA-PPD ( $s$ BPPD) PI and (b) flexible-chain $s$ BPDA-ODA ( $s$ BPOD) PI as matrices. The relationship between their morphological structures and thermophysical properties were investigated by analyzing cross-sectional SEM images and by measuring out-of-plane thermal conductivity $\left(T C_{\perp}\right)$ and in-plane coefficients of thermal expansion $\left(\mathrm{CTE}_{/ /}\right)$. The $s \mathrm{BPPD}$ chains exhibited a better polymer-filler packing behavior due to their rigid structure having small free volume. Both $T C_{\perp}$ and $\mathrm{CTE}_{/ /}$of AlN-filled $s \mathrm{BPPD}$ films showed positive deviations from theoretical predictions, whereas no such behavior was observed in AIN $/ s$ BPOD composites or in $s$ BPPD films filled with highly anisotropic hexagonal boron nitride $(\mathrm{hBN})$ flakes. The enhanced $T C_{\perp}$ and $\mathrm{CTE}_{/ /}$ observed for the $s$ BPPD composites can be attributed to a significant change in the orientational state of the PI chains; in-plane orientated $s$ BPPD chains in the pristine PI film become distorted by incorporation of isotropic AlN particles.
\end{abstract}

Keywords: Polyimide, aluminum nitride (AIN), hexagonal boron nitride (hBN), thermal diffusivity, thermal conductivity, organic-inorganic composite

\section{Introduction}

Polyimides (PIs) are a class of superengineering plastics exhibiting high thermal and chemical stability, high electric voltage and radiation resistance, and high mechanical strength [1]. Due to these outstanding properties, PIs have been widely used in electronic and aerospace industries [2]. These properties also make PIs good candidates for polymeric matrices of new inorganic/organic thermal interface materials exhibiting both high heat-resistance and enhanced thermal conductivity $(T C, \mathrm{~W} / \mathrm{m} \cdot \mathrm{K})$.

We have previously reported a detailed study on fabrication and structure-property analyses of composite films containing polyimides and various grades of hexagonal boron nitride (hBN) flakes [3]. When PI films are filled with particles having a highly anisotropic shape, a precise analysis of the effects of PI structure on TC of composites is difficult because even a slight change in the orientation of particles strongly affects the direction of heat-transport. In order to closely investigate the effect of rigidity or linearity of PI chains on the effective TC of composites, elimination or reduction of the effect of filler orientation is necessary.

In this study, we fabricated composite films using rigid-rod $s$ BPDA-PPD PI $(s \mathrm{BPPD})$ and flexible-chain $s$ BPDA-ODA PI (sBPOD) as matrices and aluminum nitride (AIN) particles having nearly isotropic shapes as fillers. The comparison of the AlN/PI with hBN/PI composites will be presented, and their structure-property relationships will be discussed in terms of the out-of-plane thermal conductivity $\left(T C_{\perp}\right)$ and the coefficient of thermal expansion in the in-plane

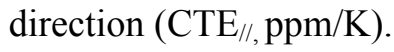

\section{Experimental}

\subsection{Materials}

AlN (Fig.1a) and hBN (Fig.1b) particles supplied by Denki Kagaku Kougyo K.K were 
vacuum-dried at $120{ }^{\circ} \mathrm{C}$ prior to use. 3,3',4,4'-Biphenyltetra carboxylic dianhydride ( $s$ BPDA, Wako Pure Chem. Ind. Ltd.) was dried at $180{ }^{\circ} \mathrm{C}$ under vacuum and used without further purification. $p$-Phenylene diamine (PPD, Wako Pure Chem. Ind. Ltd.) and 4,4'-diamino diphenylether (ODA, Kanto Chem. co., Inc) were purified by sublimation under reduced pressure. Anhydrous $N$-methyl-2-pyrrolidone (NMP, Sigma-Aldrich) was used as received. (a)

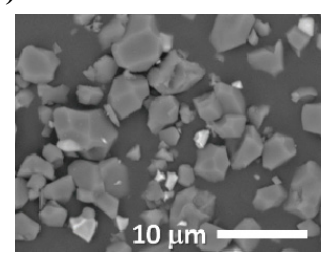

(b)

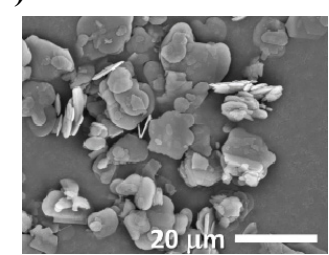

Fig. 1. SEM images of (a) AlN and (b) hBN particles

\subsection{Preparation of AIN/PI composite films}

The molecular structures of PI matrices used in this study are shown in Scheme 1.

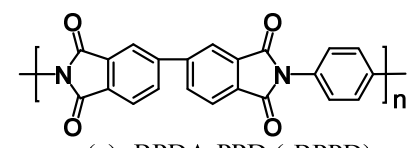

(a) $s \mathrm{BPDA}-\mathrm{PPD}(s \mathrm{BPPD})$

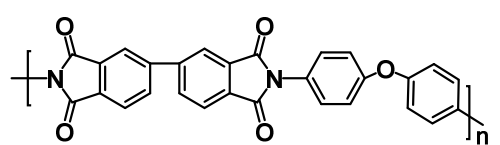

(b) $s$ BPDA-ODA $(s$ BPOD)

Scheme 1. Chemical structures of PI matrices.

AlN/PI composite films were prepared by thermal imidization of solution-processable poly (amic acid) (PAA) containing AlN particles. AlN particles were first dispersed in NMP using an ultrasonic homogenizer (US-50, Nissei Corp.), and then equimolar amounts of dianhydride and diamine were added to the mixture and stirred overnight to obtain an AlN/PAA/NMP slurry. The slurry was further mixed and defoamed using a rotation/ revolultion mixer (Thinky, ARE-310). The filler/PI composite films were prepared by spin-coating of the slurry onto silicone (Si) substrates, followed by solvent evaporation at $80{ }^{\circ} \mathrm{C}$ for $1 \mathrm{~h}$. The AlN/PAA films were peeled from the $\mathrm{Si}$ substrate in order to minimize the effect of constraint imposed by the substrate, and then thermally imidized at $350{ }^{\circ} \mathrm{C}$ for 90 min under constant nitrogen flow. hBN/PI composite films were prepared following the same procedures, except that $N, N$-dimethylacetamide was used as solvent.

\subsection{Measurements}

Cross-sections of the composite films were obtained by a JEOL SM-09020CP cross-section polisher. SEM images of the Au-coated cross-sections were observed with a Hitachi Tabletop TM-3000 microscope. The void concentration $\left(\phi_{v}\right)$ was evaluated from binary-converted SEM images using a NIH ImageJ software [4]. Out-of-plane thermal diffusivities $\left(T D_{\perp}\right)$ were measured with a temperature wave analyzer (ai-phase mobile $1 \mathrm{u},[5])$. The $T C_{\perp}$ values $[\mathrm{W} / \mathrm{m} \cdot \mathrm{K}]$ were calculated using Eqs.1-3, assuming that the density $\rho_{\mathrm{c}}\left[\mathrm{kg} / \mathrm{m}^{3}\right]$ and specific heat $C_{\mathrm{p}}$ $[\mathrm{kJ} / \mathrm{kg} \cdot \mathrm{K}]$ follows the rule of mixture. The subscripts $\mathrm{c}$, $\mathrm{f}$, and $\mathrm{m}$ represent the composite, filler, and matrix, respectively, and $V$ represents the volume fraction.

$$
\begin{array}{ll}
T C_{\perp}=T D_{\perp} \cdot C_{\mathrm{pc}} \cdot \rho_{\mathrm{c}} & \text { Eq. } 1 \\
\rho_{\mathrm{c}}=\rho_{\mathrm{f}} V+\rho_{\mathrm{m}}(1-V) & \text { Eq. } 2 \\
C_{\mathrm{pc}}=C_{\mathrm{pf}} V+C_{\mathrm{pm}}(1-V) & \text { Eq. } 3
\end{array}
$$

Coefficients of thermal expansion (CTEs) were measured using a thermal mechanical analyzer (Shimadzu TM-60) at a heating rate of $5^{\circ} \mathrm{C} / \mathrm{min}$.

\section{Results and Discussion}

\subsection{Characterization of AlN/PI films}

PI films containing 10 to $60 \mathrm{vol} \%$ of $\mathrm{AlN}$ particles were successfully prepared, and their cross-sections were obtained by argon ion beam using a cross-section polisher. In order to simplify the sample names, they will be referred to as [volume content]v-[name of filler] / [type of PI]. For example, the name for a sBPDA-ODA film containing $10 \mathrm{vol} \%$ of AlN is $10 \mathrm{v}-\mathrm{AlN} / \mathrm{sBPOD}$.

Figure 2 shows the cross-sectional SEM images of the composite films containing 30 to $60 \mathrm{vol} \%$ of AlN. These images show that the AlN particles are homogeneously distributed within the films, and no aggregation of particles was observed. This indicates that AlN particles show good affinity to both types of the PI matrices ( $s$ BPPD and $s$ BPOD) even though no surface treatment of the filler was performed. The AlN/PI films contained no apparent voids below the filler content of $30 \mathrm{vol} \%$, but voids become visible at $40 \mathrm{vol} \%$ and above. 


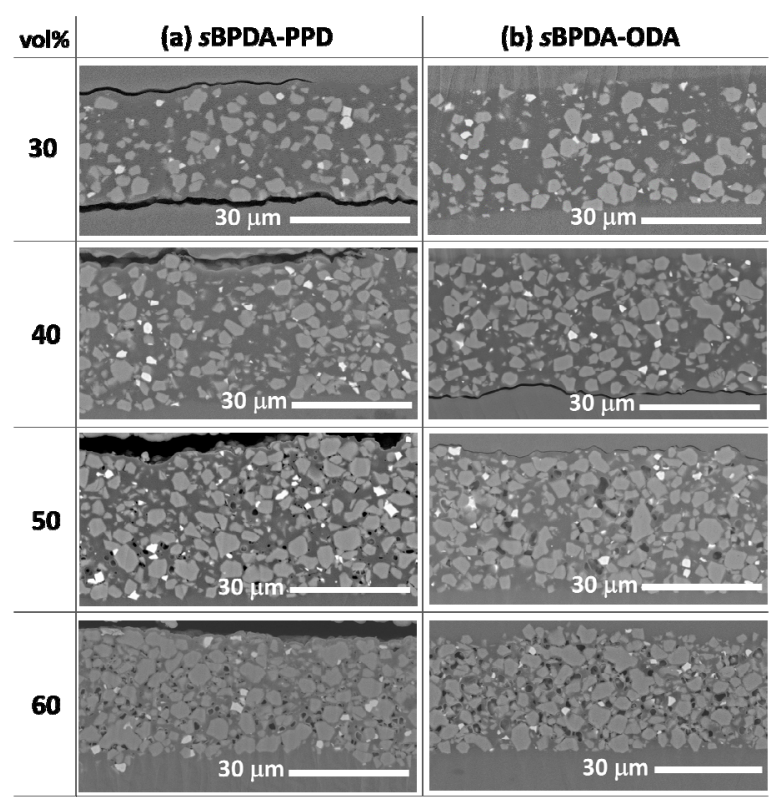

Fig. 2 Cross-sectional SEM images of AIN/PI films. (a) AlN $/ s$ BPPD and (b) AlN $/ s$ BPOD

In order to make a detailed comparison, void concentrations $\left(\phi_{v}\right)$ calculated from the SEM images are shown in Figure 3. This graph clearly shows that the $s$ BPPD composites have denser morphology than the sBPOD composites, containing smaller amounts of voids at AlN contents above $40 \mathrm{vol} \%$.

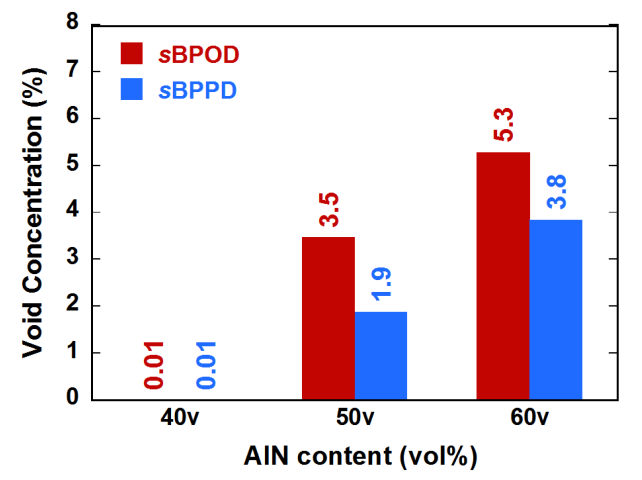

Fig. 3 The void concentration $\left(\phi_{v}\right)$ of $40 v$ to $60 \mathrm{v}-\mathrm{AlN} / \mathrm{PI}$.

The dense packing morphology of fillers and the matrix in the $s \mathrm{BPPD}$ composites can be attributed to the large packing coefficient $\left(K_{\mathrm{p}}\right)$ of the $s$ BPPD chains. $K_{\mathrm{p}}$ is defined by the ratio of van der Waals volume $\left(V_{\mathrm{wdv}}\right)$ of the repeating unit to the volume occupied by the repeating unit $\left(V_{\text {int }}\right) . V_{\text {int }}$ is the sum of $V_{\mathrm{vdw}}$ and interstitial free volume. A large $K_{\mathrm{p}}$ value therefore corresponds to small free volume. The $K_{\mathrm{p}}$ values for $s$ BPPD and $s$ BPOD have been reported as 0.6258 and 0.6018 , respectively [6]. The densities of Upilex-S and Upilex-R, which are commercial PIs having the same chemical structures as $s$ BPPD and $s$ BPOD, are reported by the manufacturer (Ube Industries) as 1.47 and 1.39, respectively. As the difference in $K_{\mathrm{p}}$ is reflected in the density of pristine PIs, the difference in $\phi_{v}$ suggests that a large $K_{\mathrm{p}}$ value also contributes to the denser packing between the matrix PI and filler particles. The same filler-polymer packing behavior has also been observed in a series of $\mathrm{hBN} / \mathrm{PI}$ composite films [7].

\subsection{Thermal conductivity of AlN/PI films}

The out-of-plane thermal conductivity $\left(T C_{\perp}\right)$ with respect to AlN content is shown in Figure 4.

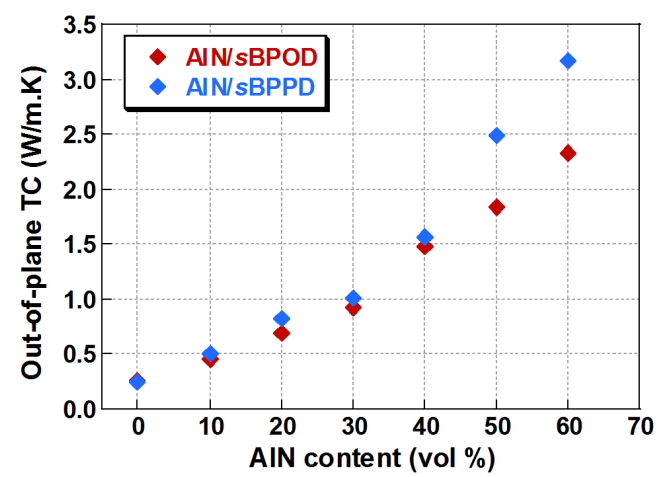

Fig.4 Out-of-plane thermal conductivities of AlN/PI films.

Both composites show a similar trend of increasing $T C_{\perp}$ at higher AlN contents. At 0 vol\%, both types of PIs have similar $T C_{\perp}$ values: 0.256 $\mathrm{W} / \mathrm{m} \cdot \mathrm{K}$ for $s \mathrm{BPOD}$ and $0.248 \mathrm{~W} / \mathrm{m} \cdot \mathrm{K}$ for $s$ BPPD. In the composite films, however, the $s$ BPPD composites exhibit larger values at all AlN contents, and the difference becomes larger as a larger amount of AlN is incorporated. The smaller $T C_{\perp}$ in $50 \mathrm{v}$ and $60 \mathrm{v}-\mathrm{AlN} / \mathrm{sBPOD}$ films may be attributed to the difference in $\phi_{v}$, but it does not explain why the voidless composite films also show the same trend.

It has been reported that the major factors contributing to $T C$ of PIs are (1) the chemical structure of the repeating unit, (2) the orientation of chains, and (3) the degree of molecular packing [8-10]. In dielectric materials, heat is transferred via quantized lattice vibrations called 'phonons'. Due to the nature of the mechanism, efficient heat propagation in polymeric materials occurs in cases when the repeating unit has high rigidity and when the packing of polymer chains is dense, both of which reduce anharmonicity of lattice vibration. For the same reason, heat is more efficiently propagated along polymer chains than from one 
chain to another. In the case of $s$ BPPD and $s$ BPOD, the rigid molecular chains of the former PI have dense interchain packing and tend to orient in the in-plane direction of the film, while those of the latter PI are oriented more isotropically due to their flexible molecular structure and looser interchain packing. Although the $T C_{\perp}$ values of pristine $s$ BPPD and $s$ BPOD are similar, they have different structural origin. The $T C_{\perp}$ value of $s \mathrm{BPPD}$ is comparable to $s \mathrm{BPOD}$ even though it has rigid and densely packed chains. This is because the $T D_{\perp}$ measurement was performed in the direction perpendicular to the orientation of $s$ BPPD chains. The $T C_{\perp}$ of $s \mathrm{BPOD}$, on the other hand, is relatively high because a larger proportion of PI chains contributes to heat propagation in the out-of-plane direction. It is confirmed by the fact that reported in-plane $T D\left(T D_{/ /}\right)$of $s$ BPPD and $s$ BPOD are approximately 5-fold and 2-fold of their $T D_{\perp}$, respectively [9].

According to the Bruggeman model [11] and other numerous theoretical models, the effective $T C$ of particulate composite largely depends on the $T C$ of the matrix, and if the matrix $T C$ increases and other conditions such as the filler concentration, the filler $T C$ and interfacial resistance are unchanged, the predicted $T C$ values for composites always increase.

For comparison, the cross-sectional SEM images of $40 \mathrm{v}-\mathrm{hBN} / \mathrm{PI}$ films are shown as examples in Figure 5, and the $T C_{\perp}$ values of $\mathrm{hBN} / \mathrm{PI}$ composite films versus the $\mathrm{hBN}$ content are plotted in Figure 6.
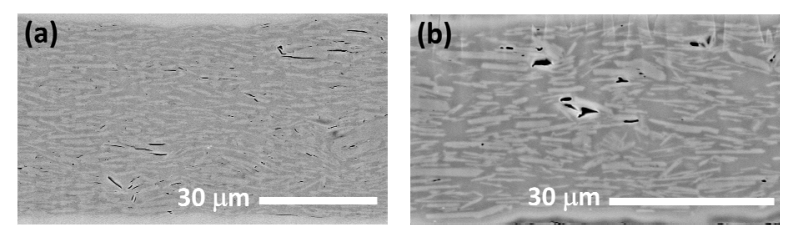

Fig.5 Cross-sectional SEM images of (a) $40 \mathrm{v}-\mathrm{hBN} / s \mathrm{BPPD}$ and $40 \mathrm{v}-\mathrm{hBN} / \mathrm{sBPOD}$.

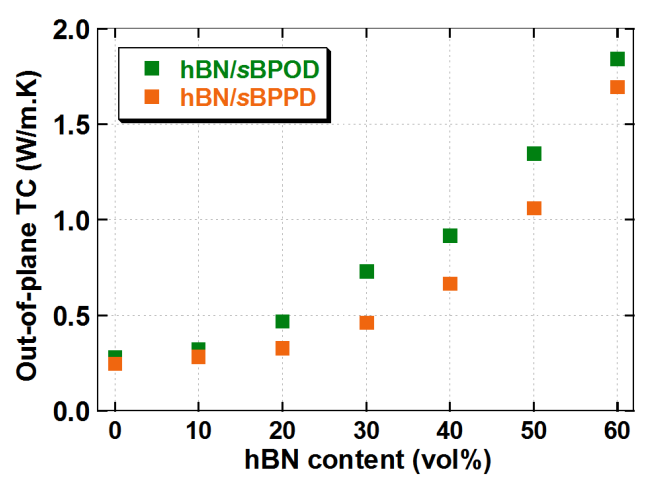

Fig.6 Out-of-plane thermal conductivities of hBN/PI films.
In Figure 5, hBN flakes are observed as thin white lines dispersed in the gray background of PI matrices, indicating that the basal planes of $\mathrm{hBN}$ are strongly oriented parallel to the film plane. Relatively small $T C_{\perp}$ values of $\mathrm{hBN}$-filled films compared to AlN-filled films is probably due to this structural character, which has higher thermal resistance along stacked $\mathrm{hBN}$ layers.

When filled with highly anisotropic hBN flakes, the composite films of $s \mathrm{BPOD}$ exhibit higher composite $T C_{\perp}$ at all $\mathrm{hBN}$ contents, even though the $\phi_{v}$ values are larger for the $s$ BPOD composites than the $s$ BPPD composites. These results reflect a contribution of a slightly higher $T C_{\perp}$ of the $s$ BPOD matrix, as predicted by the theoretical models.

Given these facts, the reason for the higher $T C_{\perp}$ of AlN/sBPPD composites can be speculated that the strong in-plane orientation of the rigid $s$ BPPD chains becomes less anisotropic by incorporation of nearly isotropic AlN partiles. A schematic image is shown in Figure 7.

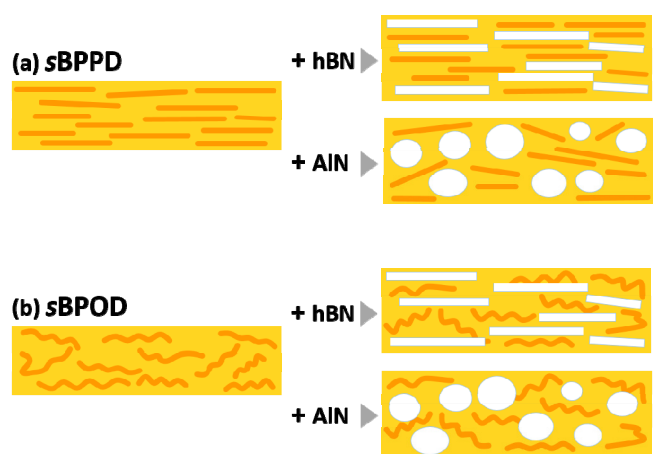

Fig.7 Schematic image of orientational relaxation of PI chains in composite films.

Incorporation of highly anisotropic hBN particles does not affect the orientation of $s$ BPPD chains because both of them tend to orient in the in-plane direction and do not interfere with each other. The presence of AlN particles, however, distorts the in-plane orientation of $s$ BPPD chains, and as a result, the contribution of heat propagation along the $s$ BPPD chains increases in the out-of-plane diretion of the films. On the other hand, the $s$ BPOD films do not show such anomalous behaviors because the flexible $s$ BPOD chains in the pristine film are oriented more isotropically and less susceptible to the presence of filler particles.

\subsection{Thermal expansion behaviors of composites}

Here, we further investigate the structure-property relationships of the AlN/PI 
composite films by examining their in-plane thermal expansion behaviors represented by CTEs. CTE is another thermophysical property that strongly reflects the structural anisotropy. It has been reported that PIs having rigid and linear main chains show smaller in-plane CTE $\left(\mathrm{CTE}_{/ /}\right)$than PIs having flexible main chains [12-15]. In the out-of-plane direction, however, rigid PIs are known to expand several tens of times more than $\mathrm{CTE}_{/ /}[16]$. The CTE values of the two types of PI films are shown in Table 1 . The $\mathrm{CTE}_{/ /}$were the values measured in this study by TMA, and the $\mathrm{CTE}_{\perp}$ are literature values measured by near-infrared optical interferometry [16].

Table 1. In-plane and out-of-plane CTEs of PIs

\begin{tabular}{ccc}
$\mathrm{PI}$ & $\mathrm{CTE}_{/ /}$ & $\mathrm{CTE}_{\perp}[16]$ \\
\hline \hline$s$ BPDA-PPD & 6.8 & 133.2 \\
\hline$s$ BPDA-ODA & 44.7 & 50.2
\end{tabular}

Figure 8 shows the TMA curves of pristine PI films and 10v-AIN/PI films. The thermal expansion behaviors were almost linear in all samples within the range of 80 and $180{ }^{\circ} \mathrm{C}$. It is expected that incorporation of AlN particles reduces the composite $\mathrm{CTE}_{/ /}$because AlN has a $\mathrm{CTE}$ as small as $4.5 \mathrm{ppm} / \mathrm{K}$. However, the in-plane thermal expansion of the $s$ BPPD films increased by an addition of $10 \mathrm{vol} \%$ of AlN.

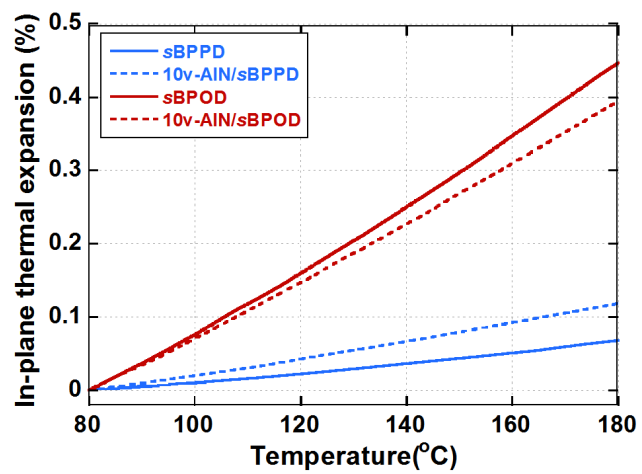

Fig.8 TMA curves of pristine PIs and 10v-AIN/PIs

The $\mathrm{CTE}_{/ /}$values estimated from TMA curves of AlN/PI samples are plotted in Figure 9. The $\mathrm{CTE}_{/ / \mathrm{S}}$ of hBN/PI films are also plotted in Figure 10 for comparison. The dotted lines in Figures 9 and 10 show the $\mathrm{CTE}_{/ /}$values estimated using the rule of mixture, defined by $\mathrm{CTE}_{/ /(\mathrm{c})}=\mathrm{CTE}_{(\mathrm{f})} V+\mathrm{CTE}_{/ /(\mathrm{m})}(1$ $-V)$. A clear difference was observed between the two types of composite films; in Figure 8, the $\mathrm{CTE}_{/ /}$of $s \mathrm{BPOD}$ composites decreases almost linearly as the AlN content increases, while that of $s$ BPPD composites initially increases and then decreases, showing a maximum at $20 \mathrm{vol} \%$ AlN content. In contrast, the $\mathrm{CTE}_{/ /}$of PIs filled with $\mathrm{hBN}$ particles approachs that of pristine $\mathrm{hBN}$ $\left(\mathrm{CTE}_{a \text {-axis }}=-2.9 \mathrm{ppm} / \mathrm{K}\right)$ as the filler content increases.

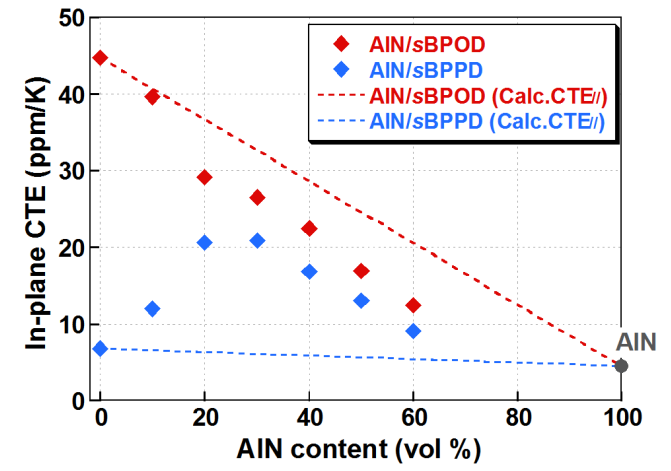

Fig.9 In-plane CTEs of AlN/PI films $\left(80-180{ }^{\circ} \mathrm{C}\right)$.

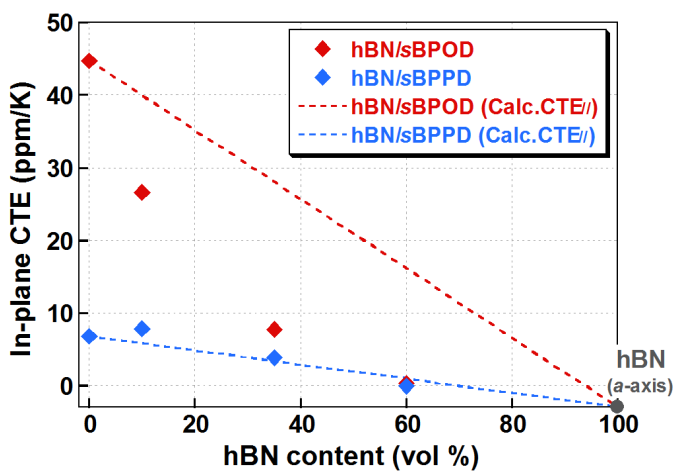

Fig.10 In-plane CTEs of hBN/PI films $\left(80-180^{\circ} \mathrm{C}\right)$.

Again, we attribute this trend to the orientational relaxation of $s \mathrm{BPPD}$ chains induced by incorporation of AIN particles having nearly isotropic shapes. It can be assumed that the contribution of interchain expansion increases with a reduction in the degree of in-plane orientation, leading to an increase in $\mathrm{CTE}_{/ /}$.

Assuming that the thermal expansion behavior of AlN-filled films obeys the rule of mixture, the $\mathrm{CTE}_{/ /}$of matrix $s \mathrm{BPPD}$ under the presence of AIN particles can be approximated (Figure 11). The estimated CTE of the $s$ BPPD matrix has a maximum at $30 \mathrm{vol} \%$. It can be inferred from the plot that an addition of $30 \mathrm{vol} \%$ of this particular type of filler exerts the highest efficiency in distorting the orientation of $s$ BPPD chains, whereas further addition of the filler beyond this volume content results in just a slight increase in in-plane oriented chains. Furthermore, it also demonstrates that an addition of only $10 \mathrm{vol} \%$ of AlN effectively alters the composite properties. A 
more precise and reliable method for determining the physical properties of the matrices in the presence of filler remains to be established and is currently under investigation.

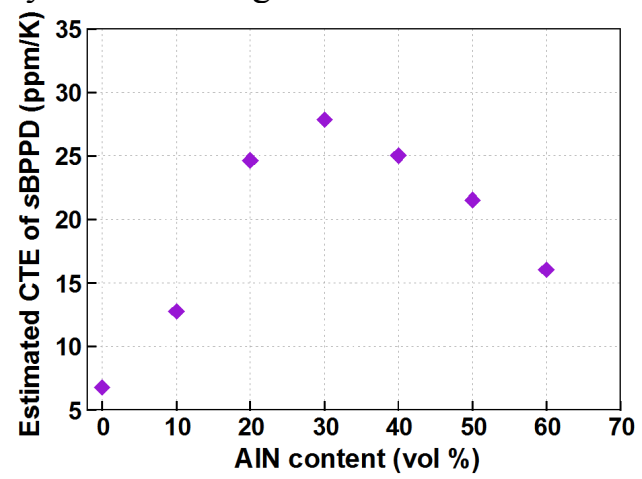

Fig.11 Estimated CTE of $s$ BPPD matrix in the presence of AlN particles

\section{Conclusion}

A series of composite films containing $\mu \mathrm{m}$-sized particles of aluminum nitride (AIN) having nearly isotropic shapes were prepared using two types of polyimide (PI) matrices: (a) $s$ BPDA-PPD ( $s$ BPPD) and (b) $s$ BPDA-ODA ( $s$ BPOD), having a rigid and flexible molecular structures, respectively. The composite films were successfully fabricated from solution-processable slurry of poly(amic acid) and AlN particles. The AlN particles were homogeneously dispersed within the films without any noticeable aggregations. Moreover, a good interfacial affinity between $\mathrm{AlN}$ and PI was observed, although no surface treatment of the filler was performed. It was revealed by image analysis of cross-sectional SEM images that rigid chains of $s$ BPPD are favorable in terms of filler-PI packing, resulting in a significantly small void concentration $\phi_{\mathrm{v}}$ at high AlN contents.

The out-of-plane thermal conductivities $\left(T C_{\perp}\right)$ of AlN $/ s$ BPPD composites exhibited anomalously larger values than those predicted on the basis of effective medium approximations. Such a phenomenon was not observed in the PI films filled with highly anisotropic hexagonal boron nitride $(\mathrm{hBN})$ flakes. It can be attributed to the change in the orientational state of $s$ BPPD chains caused by the presence of AlN particles, in which incorporation of AIN particles distorts in-plane orientation of the $s$ BPPD chains, increasing the contribution of heat transport along the chains in the out-of-plane direction of the composite films.

The in-plane coefficients of thermal expansion $\left(\mathrm{CTE}_{/ / \mathrm{s}}\right)$ of AlN/sBPOD composites decreased almost linearly with increasing AlN content, while those of the AlN/sBPPD composites initially increased and then decreased with a maximum at $30 \mathrm{vol} \%$ AlN, showing good agreement with the result of $T C_{\perp}$ measurement.

These results evidently demonstrate that combining nearly isotropic fillers and highly anisotropic polymer matrix is an effective strategy for tuning the composite properties. Future work is planned on a more comprehensive and quantitative understanding of filler-polymer interaction.

\section{Acknowledgements}

We would like to thank the following individuals at Tokyo Institute of Technology: Dr. T. Hashimoto and Dr. J. Morikawa for their help in measurement of thermal diffusivity, Dr. S. Asai for his advice in image analysis, Dr. T. Isobe for his help in separation and handling of ceramic particles, and Mr. J. Koki and Ms. Y. Ohtsuka (Center for Advanced Materials Analysis) for their support in sample preparation using a cross-section polisher. We would also like to acknowledge Dr. T. Yamagata and Dr. K. Miyata (Denki Kagaku Kougyo K.K.) for helpful discussions and their continuous support.

\section{References}

1. K.L. Mittal, 'Polyimides: Synthesis, Characterization, and Applications', Plenum Publishing Corp. (1984).

2. M. Ghosh, 'Polyimides: Fundamentals and Applications', CRC Press (1996).

3. M. Tanimoto, T. Yamagata, K. Miyata, and S. Ando. ACS Appl. Mater. Interfaces, 10 (2013) 4374.

4. C. Schneider, W. Rasband, and K. Eliceiri. Nature Methods, 9 (2012) 671.

5. T. Hashimoto, J, Morikawa, T. Kurihara, and T. Tsuji. Thermochim. Acta, 305 (1997) 151.

6. Y. Terui, S. Matsuda, and S. Ando. J. Polym. Sci. Part B: Polym. Phys. 43 (2005) 2109.

7. M. Tanimoto and S. Ando. Manuscript submitted for publication (2014).

8. K. Kurabayashi and K. Goodson. J. Appl. Phys. 86 (1999) 1925.

9. J. Morikawa and T. Hashimoto. J. Appl. Phys. 105 (2009) 113506-1-9.

10.D. Yorifuji and S. Ando. Macromolecules, 43 (2010) 7583 .

11.D. A. G. Bruggeman. Annalen Der Physik, 25 (1936) 645.

12. S. Numata, S. Oohara, J. Imaizumi and N. Kinjo. Polym. J., 17 (1985) 981.

13. S. Numata, S. Oohara, K. Fujisaki, J. Imaizumi and N. Kinjo. J. Appl. Polym. Sci., 31 (1986) 101.

14. S. Numata, K. Fujisaki and N. Kinjo. Polymer, 28 (1987) 2283.

15. M. Hasegawa and S. Horii, Polym. J., 39 (2007) 610.

16. K. Sekiguchi and S. Ando, Polymer Prep. Jpn., 60 (2011) 665 . 